\title{
A Chosen Plaintext Attack of the 16-round Khufu Cryptosystem
}

\author{
Henri Gilbert and Pascal Chauvaud \\ France Télécom-CNET \\ PAA-TSA-SRC \\ 38-40 Rue du Général Leclerc, 92131 Issy-les-Moulineaux \\ France
}

\begin{abstract}
In 1990, Merkle proposed two fast software encryption functions, Khafre and Khufu, as possible replacements for DES [1]. In 1991, Biham and Shamir applied their differential cryptanalysis technique to Khafre [2], and obtained an efficient attack of the 16-round version and some bounds on the 24-round version. However, these attacks take advantage of the fact that the S-boxes used for Khafre are public; they cannot be applied to Khufu, which uses secret S-boxes, and no attack of Khufu has been proposed so far. In this paper, we present a chosen plaintext attack of the 16-round version of Khufu, which is based on differential properties of this algorithm. The derivation of first information concerning the secret key requires about $2^{31}$ chosen plaintexts and $2^{31}$ operations. Our estimate of the resources required for breaking the entire scheme is about $2^{43}$ chosen plaintexts and about $2^{43}$ operations.
\end{abstract}

\section{Description of Khufu}

Khufu is an iterated blockcipher with a 64-bit blocksize. The keys used in the 16-round version (the single one considered in this paper) are the following :

- four 32-bit words K1, K2, K3, K4, used before the first round and after the last round (initial and final xors);

- four secret permutations $p_{0}, p_{1}, p_{2}$ and $p_{3}$ of the [0..255] set (the columns of the first S-box introduced in [1]), which provide the functions used in rounds 1 to 8 ;

- four secret permutations $q_{0}, q_{1}, q_{2}$ and $q_{3}$ of the $[0.255]$ set (the columns of the second S-box introduced in [1]), which provide the functions used in rounds 9 to 16 .

The 16-round version of Khufu is depicted in Figure 1, which represents the encryption of one 64-bit block consisting of two 4-byte halves $L=\left(l_{0}, l_{1}, l_{2}, l_{3}\right)$ and $R=\left(r_{0}, r_{1}, r_{2}, r_{3}\right)$. We are using the following notations :

$-\pi_{i}(i=0$ to 3$)$ denotes the projection:

$\pi_{i}: \quad[0.255]^{4} \rightarrow[0.255]$

$\left(x_{0}, x_{1}, x_{2}, x_{3}\right) \mapsto x_{i}$ 
- $\left[p_{i_{0}}, p_{i_{1}}, p_{i_{2}}, p_{i_{3}}\right]$ (where $\left(i_{0}, i_{1}, i_{2}, i_{3}\right)$ is a circular permutation of $(0,1,2,3)$ ) denotes an $\mathrm{S}$-box the columus of which are provided by the $p_{i_{0}}, p_{i_{1}}, p_{i_{2}}$ and $p_{i_{3}}$ permutations:

$$
\begin{aligned}
{\left[p_{i_{0}}, p_{i_{1}}, p_{i_{2}}, p_{i_{3}}\right]:[0 . .255] } & \rightarrow[0.255]^{4} \\
x & \mapsto\left(p_{i_{0}}[x], p_{i_{1}}[x], p_{i_{2}}[x], p_{i_{3}}[x]\right)
\end{aligned}
$$

The representation of Figure 1 is slightly different from the one provided in [1]: in order to avoid swapping the right and left halves $\mathrm{R}$ and $\mathrm{L}$ and rotating the $\mathrm{R}$. half at each round, we are using a different round function at each round. It is however easy to check that both representations are strictly equivalent. For a more detailed presentation of the Khufu algorithm, see [1].
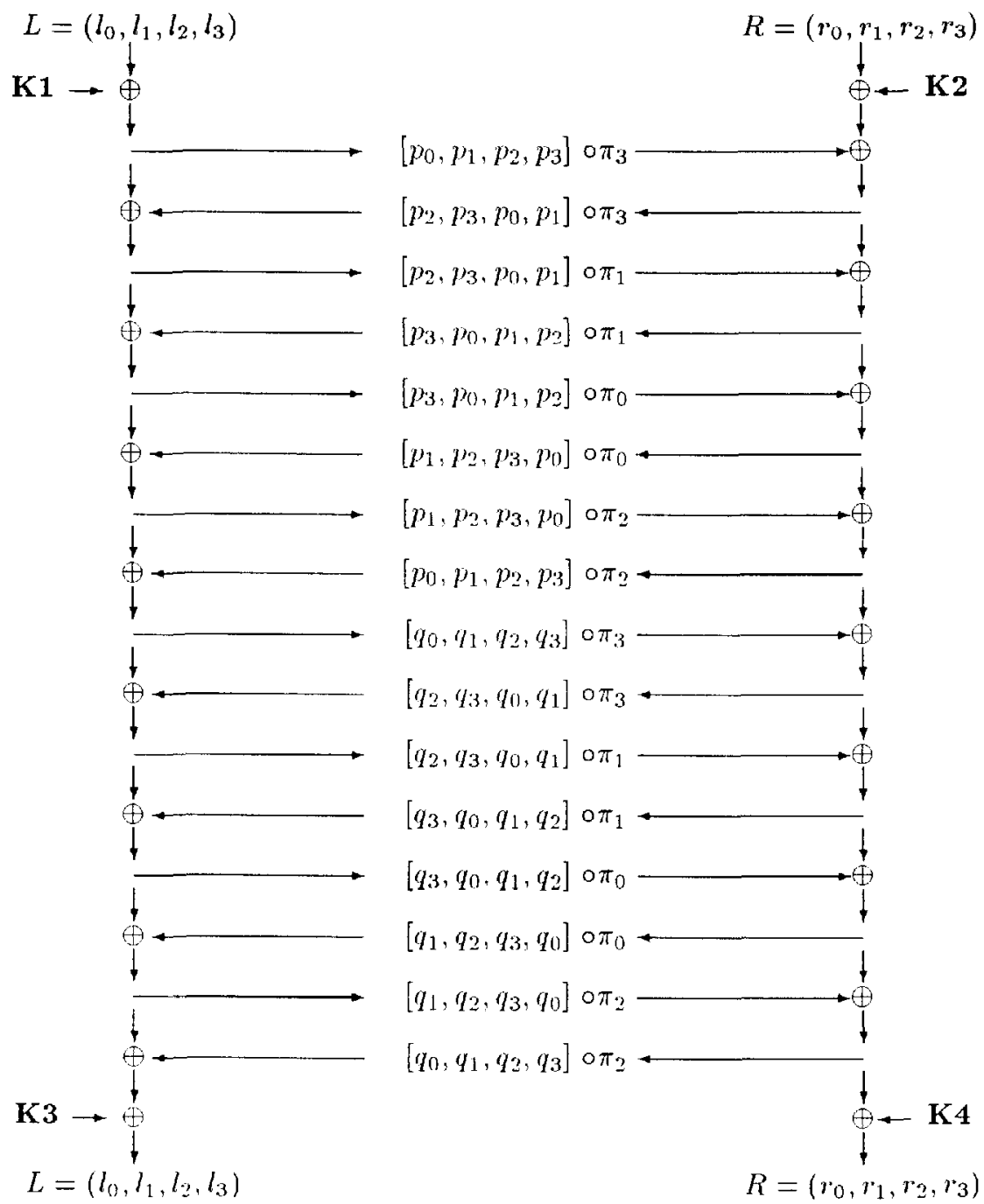

Fig. 1. The 16-round version of Khufu 


\section{Differential properties used in our attack}

At the various steps of our attack, sets of $n$ (P, $\left.\mathrm{P}^{\prime}\right)$ pairs of 64 -bit plaintext blocks are considered. In practice, these sets are defined by a combination of conditions on :

- the plaintext difference $\mathrm{P} \oplus \mathrm{P}^{\prime}$;

- some bytes of $P$.

Let $S$ be such a set of $n$ (P, $\left.\mathrm{P}^{\prime}\right)$ pairs. After $r$ rounds (where $r \in[0, \ldots, 16]$ ), a $\left(\mathrm{P}, \mathrm{P}^{\prime}\right)$ pair provides a $\left(C[r], C^{\prime}[r]\right)$ pair of intermediate blocks, of xor value : $\Delta[r]=C[r] \oplus C^{\prime}[r]$. We denote by $\Delta S[r]$ the set of $\Delta[r]$ values derived from the $n\left(\mathrm{P}, \mathrm{P}^{\prime}\right)$ pairs in $S$ after $r$ rounds.

All the differential properties we are using in our attack can be expressed in terms of the cardinal $|\Delta S[r]|$ of the $\Delta S[r]$ set of the distinct difference values after $r$ rounds for an appropriately selected set $S$ of plaintext pairs.

The following Proposition will be useful for the initial step of our attack:

Proposition 1. Let $\lambda_{1}, \lambda_{3}$ and $\rho_{3}$ be arbitrary constants in [0.25.5].

For every $\alpha \in] 0 . .255]$, there exist four constants $\delta_{0}, \delta_{1}, \delta_{2}$ and $\beta \in[0.255]$ such that, if $S_{\lambda_{1}, \lambda_{3}, \beta_{3}, \alpha}$ denotes the following set of plaintext pairs:

$S_{\lambda_{1}, \lambda_{3}, \rho_{3}, \alpha}=\left\{\left((L, R) ;\left(L^{\prime}, R^{\prime}\right)\right) \mid L \oplus L^{\prime}=(0, \alpha, 0,0) ; R \oplus R^{\prime}=\left(\delta_{0}, \delta_{1}, \delta_{2}, 0\right)\right.$;

$\left.l_{1}=\lambda_{1} ; l_{3}=\lambda_{3} ; r_{3}=\rho_{3}\right\}$

then $\Delta S_{\lambda_{1}, \lambda_{3}, \rho_{3}, \alpha}[9]=((0, \alpha, 0,0),(0,0,0, \beta))$,

$i$. e. the difference for the various pairs of the $S_{\lambda_{1}, \lambda_{3}, \beta_{3}, \alpha}$ set is constant after 9 rounds.

Proof : After two rounds, the $\left(l_{1}, l_{1}^{\prime}\right)$ pair is fixed and equal to the two constant bytes :

$c_{1}=\lambda_{1} \oplus K 1_{1} \oplus p_{3}\left[\beta_{3} \oplus K 2_{3} \oplus p_{3}\left[\lambda_{3} \oplus K 1_{3}\right)\right] ; c_{1}^{\prime}=c_{1} \oplus \alpha$

furthermore, these $l_{1}$ and $l_{1}^{\prime}$ values act as imputs to the $\left[p_{2}, p_{3}, p_{0}, p_{1}\right]$ S-box at round 3 . It suffices to select $\delta_{0}, \delta_{1}, \delta_{2}$ as to "compensate" the difference between the two $\left[p_{2}, p_{3}, p_{0}, p_{1}\right]$ outputs to obtain a constant xor value for the six subsequent rounds. The encryption of a $S_{\lambda_{1}, \lambda_{3}, \beta_{3}, \alpha}$ pair is depicted in Figure 2 (where the difference value at each round is provided).

The $\delta_{0}, \delta_{1}, \delta_{2}$ and $\beta$ constants are given by the relation :

(1) : $\left(\delta_{0}, \delta_{1}, \delta_{2}, \beta\right)=\left[p_{2}, p_{3}, p_{0}, p_{1}\right]\left[c_{1}\right] \oplus\left[p_{2}, p_{3}, p_{0}, p_{1}\right]\left[c_{1}^{\prime}\right]$ where $c_{1}$ and $c_{1}^{\prime}$ are defined above. 


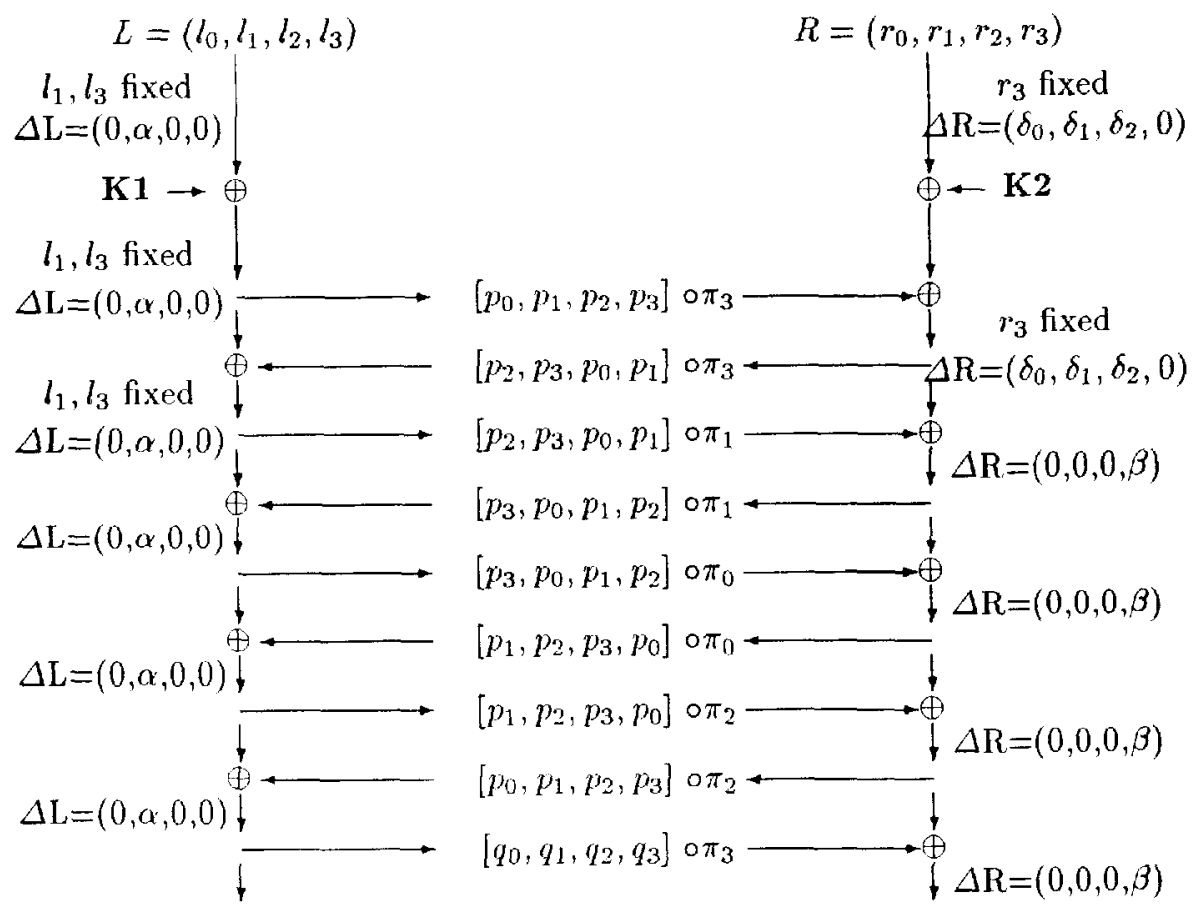

Fig. 2. Encryption of an $S_{\lambda_{1}, \lambda_{3}, \rho_{3}, \alpha}$ pair

The next proposition, which is very similar to Proposition 1, will be useful for the subsequent steps of our attack.

Proposition 2. Let $\lambda_{3}$ be arbitrary constant $\in[0 . .255]$. For every $\left.\left.\alpha \in\right] 0.255\right]$, there exist four constants $\delta_{0}, \delta_{1}, \delta_{2}$ and $\delta_{3} \in[0 . .255]$ such that, if $S_{\lambda_{3}, \alpha}$ denotes the following set of plaintext pairs:

$S_{\lambda_{3}, \alpha}=\left\{\left((L, R) ;\left(L^{\prime}, R^{\prime}\right)\right) \mid L \oplus L^{\prime}=(0,0,0, \alpha) ; R \oplus R^{\prime}=\left(\delta_{0}, \delta_{1}, \delta_{2}, \delta_{3}\right) ; l_{3}=\lambda_{3}\right\}$ then $\Delta S_{\lambda_{3}, \alpha}[8]=\{((0,0,0, \alpha),(0,0,0,0))\}$, i. e. the difference for the various pairs of the $S_{\lambda_{3}, \mathrm{ex}}$ set is constant after 8 rounds.

Proof: The proof is similar to l.he proof of Proposition 1. The $l_{3}$ and $l_{3}^{\prime}$ inputs to the $\left[p_{0}, p_{1}, p_{2}, p_{3}\right] S$-box in the first round are the constant bytes:

$c_{3}=\lambda_{3} \oplus K 1_{3}$ and $c_{3}^{\prime}=c_{3} \oplus \alpha$.

It suffices to take for $\delta_{0}, \delta_{1}, \delta_{2}$ and $\delta_{3}$ the constants given by the relation :

(2) : $\left(\delta_{0}, \delta_{1}, \delta_{2}, \delta_{3}\right)=\left[p_{0}, p_{1}, p_{2}, p_{3}\right]\left[c_{3}\right] \oplus\left[p_{2}, p_{3}, p_{0}, p_{1}\right]\left[c_{3}^{\prime}\right]$ to obtain the announced result. 
The next proposition essentially states that for a given set of plaintext pairs, there are some bounds limiting the increase of the number of distinct difference values after $r$ rounds. When used in conjonction with Propositions 1 and 2, Proposition 3 provides non-trivial differential properties for the whole 16-round algorithm.

Proposition 3. If $S$ is a set of $n$ ( $\left.P, P^{\prime}\right)$ plaintext pairs and if $\Delta S[r]$ is defined as above, then:

$\forall r \in[0 . .15],|\Delta S[r+1]| \leq 128 \times|\Delta S[r]|$

Proof : Let $\Delta[r+1]$ be a $\Delta S[r+1]$ value. There exists a (P, $\mathrm{P}^{\prime}$ ) pair in $S$ leading to the $\Delta[r+1]$ xor value after $r+1$ rounds. Let us denote by $\Delta[r]$ the xor value for this pair after $r$ rounds, and by $\delta[r]$ the byte of $\Delta[r]$ which position is picked up as an input to the $S$-box used in round $(r+1)$; by $a$ the input to the S-box of round $(r+1)$ in the encryption of $P$.

Depending on the parity of $r$, we have either :

$\left(^{*}\right): \Delta[r+1]=\Delta[r] \oplus((S b o x[a] \oplus S b o x[a \oplus \delta[r]]),(0,0,0,0))$

or

$(* *): \Delta[r+1]=\Delta[r] \oplus((0,0,0,0),(S b \circ x[a] \oplus S b o x[a \oplus \delta[r]]))$.

Moreover, $\Delta[r] \in \Delta S[r]$ by definition.

The results now follow from the obvious property that, for a fixed value of $\Delta[r]$ (which determines a fixed value for $\delta[r]$ ), we have :

$|\{S b o x[a] \oplus S b o x[a \oplus \delta[r]] \mid a \in[0 . .255]\}| \leq 128$.

\section{An attack of the 16-round Khufu}

In this Section, we describe how to use the differential properties presented above for deriving the secret key (i.e. four 32 -bit words and 8 permutations of the $[0 . .255]$ set) from about $2^{43}$ chosen plaintexts.

The proposed attack is divided in four main steps. Only the first Step (the derivation of first information about the secret key) is developed in some detail. The purpose of the description of the subsequent steps is only to give some evidence that the information obtained at Step 1 can be used for breaking the entire scheme.

Step 1 is based on Propositions 1 and 3. We are using the notation of Proposition 1. We fix four arbitrary constants $\lambda_{1}, \lambda_{3}$ and $\rho_{3}$ and $\alpha \neq 0$ in $[0.25 .5]$.

The purpose of Step 1 is to find the three corresponding bytes $\delta_{0}, \delta_{1}, \delta_{2}$.

We select a fixed arbitrary byte $\lambda_{2}$. For 64 different values of $l_{0}$ (for instance the 0 to 63 values) we perform the following computations : 
- We encrypt the $\mathrm{X}_{l_{0}}$ set of $2^{24}$ plaintext values $\left(\left(l_{0}, \lambda_{1}, \lambda_{2}, \lambda_{3}\right),\left(r_{0}, r_{1}, r_{2}, \rho_{3}\right)\right)$, where $\left(r_{0}, r_{1}, r_{2}\right) \in[0 . .255]^{3}$, thus obtaining $2^{24}$ encrypted values $\left(\left(l_{0}, l_{1}, l_{2}, l_{3}\right),\left(r r_{0}, r r_{1}, r r_{2}, r r_{3}\right)\right)$;

- We encrypt the $\mathrm{X}_{l_{0}}^{\prime}$ set of $2^{24}$ plaintext values $\left(\left(l_{0}, \lambda_{1} \oplus \alpha, \lambda_{2}, \lambda_{3}\right),\left(r_{0}^{\prime}, r_{1}^{\prime}, r_{2}^{\prime}, \rho_{3}\right)\right)$ where $\left(r_{0}^{\prime}, r_{1}^{\prime}, r_{2}^{\prime}\right) \in[0.255]^{3}$; thus obtaining $2^{24}$ encrypted values $\left(\left(l l_{0}^{\prime}, l_{1}^{\prime}, l_{2}^{\prime}, l_{3}^{\prime}\right),\left(r r_{0}^{\prime}, r r_{1}^{\prime}, r r_{2}^{\prime}, r r_{3}^{\prime}\right)\right)$;

- We now want to find all the (P,P') pairs in $\left(\mathrm{X}_{l_{0}} \times \mathrm{X}_{l_{0}}^{\prime}\right)$ such that $l_{2}=$ $l_{2}^{\prime} ; r r_{0}=r r_{0}^{\prime} ; r r_{2}=r r_{2}^{\prime}$ (i.e. we want to filter those pairs of $\left(\mathrm{X}_{l_{0}} \times \mathrm{X}_{l_{0}}\right)$ for which the inputs and outputs of the $S$-boxes in the three last rounds of the encryption are equal), and group these pairs according to the $\left(r_{0} \oplus r_{0}^{\prime}, r_{1} \oplus r_{1}^{\prime}, r_{2} \oplus r_{2}^{\prime}\right)$ difference value. This can be done efficiently, in about $2^{25}$ operations in average, as follows :

- we group the $X_{l_{0}}$ plaintexts according to the $\left(l_{2}, r r_{0}, r r_{2}\right)$ value, thus obtaining a list of $\mathrm{X}_{l_{0}}$ plaintexts of average size 1 for each $\left(H_{2}, r r_{0}, r r_{2}\right)$ triplet;

- we group the $\mathrm{X}_{l_{0}}^{\prime}$ plaintexts according to the $\left(l_{2}^{\prime}, r r_{0}^{\prime}, r r_{2}^{\prime}\right)$ value, thus obtaining a list of $\mathrm{X}_{l_{0}}^{\prime}$ plaintexts of average size 1 for each $\left(l l_{2}^{\prime}, r r_{0}^{\prime}, r r_{2}^{\prime}\right)$ triplet;

- for each $\left(l l_{3}, r r_{0}, r r_{2}\right)$, we consider all the ( $\left.P, P^{\prime}\right)$ pairs in the crossproduct of the corresponding lists of $\mathrm{X}_{l_{0}}$ and $\mathrm{X}_{i_{0}}$ (average number of pairs : 1);

- we group the (P, $\left.\mathrm{P}^{\prime}\right)$ pairs oblained as above according to the $\left(r_{0} \oplus r_{0}^{\prime}, r_{1} \oplus r_{1}^{\prime}, r_{2} \oplus\right.$ $\left.r_{2}^{\prime}\right)$ difference, thus obtaining a list of average size 1 for each $\left(r_{0} \oplus r_{0}^{\prime}, r_{1} \oplus r_{1}^{\prime}, r_{2} \oplus\right.$ $r_{2}^{\prime}$ ) value.

For each $\left(r_{0} \oplus r_{0}^{\prime}, r_{1} \oplus r_{1}^{\prime}, r_{2} \oplus r_{2}^{\prime}\right)$ triplet, we thus obtain, by merging the lists obtained for each $l_{0}$ value, a list $S$ of 64 (P,P') pairs in average. Let us consider the $\Delta S$ set of output xors for the $\left(P, P^{\prime}\right)$ pairs contajned in such a list :

\section{Claim :}

(i) : If $\left(r_{0} \oplus r_{0}^{\prime}, r_{1} \oplus r_{1}^{\prime}, r_{2} \oplus r_{-1}^{\prime}\right)=\left(\delta_{0}, \delta_{1}, \delta_{2}\right)$ then $|\Delta S| \leq 16$

(ii) : If $\left(r_{0} \oplus r_{0}^{\prime}, r_{1} \oplus r_{1}^{\prime}, r_{2} \oplus r_{2}^{\prime}\right) \neq\left(\delta_{0}, \delta_{1}, \delta_{2}\right)$ then $|\Delta S| \approx 64$

Proof (heuristic arguments) : In the first case, $S$ is a subset of the $S_{\lambda_{1}, \lambda_{3}, \rho_{3}, \alpha}$ considered in Proposition 1. Moreover, $\Delta S=\Delta S[13]$, because the three last rounds have no effect on the output xor of an $S$ pair and $|\Delta S[13]| \leq\left|\Delta S_{\lambda_{1}, \lambda_{3}, \rho_{3}, \alpha}[13]\right| / 2^{24}$ because $\Delta S[13]$ contains only $\Delta S_{\lambda_{1}, \lambda_{3}, \rho_{3}, \alpha}[13]$ elements $\left(\left(\delta_{l_{0}}, \delta_{l_{1}}, \delta_{l_{2}}, \delta_{l_{3}}\right)\right.$, $\left.\left(\delta_{r_{0}}, \delta_{r_{1}}, \delta_{r_{2}}, \delta_{r_{3}}\right)\right)$ such that $\delta_{t_{2}}=0$ and $\delta_{r_{0}}=0$ and $\delta_{r_{2}}=0$.

The first part of the claim now results from :

$\left|\Delta S_{\lambda_{1}, \lambda_{3}, r_{3}, \alpha}[9]\right|=1$ (by Proposition 1)

and

$\left|\Delta S_{\lambda_{1}, \lambda_{3}, \rho_{3}, \alpha}[13]\right| \leq 128^{4}\left|\Delta S_{\lambda_{1}, \lambda_{3}, \rho_{3}, \alpha}[9]\right|$ (by Proposition 3).

The second part of the claim follows from the assumption that in other cases, 
the final xor values for pairs in the $S$ set simply behave as random elements of the set of $\left(\left(\delta_{l_{0}}, \delta_{l_{1}}, \delta_{l_{2}}, \delta_{l_{3}}\right),\left(\delta_{r_{0}}, \delta_{r_{1}}, \delta_{r_{2}}, \delta_{r_{3}}\right)\right)$ differences such that $\delta_{l_{2}}=0$ and $\delta_{r_{0}}=0$ and $\delta_{r_{2}}=0$.

The above claim was confirmed by computer experimcnts, where $(i)$ was checked. Because of the difference between the behaviours (i) and (ii), the $\left(\delta_{0}, \delta_{1}, \delta_{2}\right)$ triplet can be detected, as the one leading to a $\Delta S$ set of output xors of size less than 16 .

Step 1 requires the encryption of about $2^{31}$ chosen plaintext blocks, and about $2^{31}$ operations.

Step 2 : we repeat Step 1 for all possible $\alpha \neq 0$ values (without modifying the $\lambda_{1}, \lambda_{3}$ and $\rho_{3}$ constants). We are using the notation of Proposition $1: c_{1}$ denotes the common input to the S-box of round 3 for all the plaintexts of the various $\mathrm{X}_{l_{0}}$ sets.

For every $\alpha$, we obtain the 24-bit word :

$\left(\delta_{0}, \delta_{1}, \delta_{2}\right)(\alpha)=\left[p_{2}, p_{3}, p_{0}\right]\left[c_{1}\right] \oplus\left[p_{2}, p_{3}, p_{0}\right]\left[c_{1} \oplus \alpha\right]$.

In summary, after Step 2 , the $p_{2}, p_{3}$ and $p_{0}$ permutations are entirely determined, up to the four unknown bytes $c_{1}, p_{2}\left[c_{1}\right], p_{3}\left[c_{1}\right]$ and $p_{0}\left[c_{1}\right]$. Steps 1 and 2 require to encrypt about $2^{39}$ plaintext blocks, and the computational cost of steps 1 and 2 is about $2^{39}$ operations.

Step 3 is based on Propositions 2 and 3. We fix an arbitrary constant $\lambda_{3} \epsilon$ [0..255]. We are using the notations of Proposition 2. The purpose of Step 3 is to find for every $\alpha \in[1 . .255]$ the four corresponding bytes $\delta_{0}, \delta_{1}, \delta_{2}$ and $\delta_{3}$, such that:

$\left(\delta_{0}, \delta_{1}, \delta_{2}, \delta_{3}\right)(\alpha)=\left[p_{0}, p_{1}, p_{2}, p_{3}\right]\left[c_{3}\right] \oplus\left[p_{0}, p_{1}, p_{2}, p_{3}\right]\left[c_{3} \oplus \alpha\right]$.

We are using the fact that since $\left[p_{2}, p_{3}, p_{0}\right]$ is known up to the unknown bytes $c_{1}, p_{2}\left[c_{1}\right], p_{3}\left[c_{1}\right]$ and $p_{0}\left[c_{1}\right], \delta_{0}, \delta_{2}$, and $\delta_{3}$ ase known up to the single unknown byte $c_{1} \oplus c_{3}$ : there are only 256 possible values for the $\left(\delta_{0}, \delta_{2}, \delta_{3}\right)$ triplet.

Step 3 is divided in two substeps, numbered 3.1 and 3.2 .

Substep 3.1: We want to find $\left(\delta_{0}, \delta_{1}, \delta_{2}, \delta_{3}\right)$ for a first fixed $\alpha \neq 0$ value. We are doing an exhantive search on the $c_{1} \oplus c_{3}$ byte. For each $c_{1} \oplus c_{3}$ assumption, the candiclate values $\delta_{0}, \delta_{2}$ and $\delta_{3}$ are deternined, and we efficiently test the 256 candidate $\delta_{1}$ values by a method close to the one of Step 1. For that purpose, we select fixed arbitrary bytes $\rho_{0}, \rho_{2}$ and $\rho_{3}$; for $2^{24}$ different values of the $\left(l_{0}, l_{1}, l_{2}\right)$ triplet, we perform the following computations:

- We encrypt the $X_{l_{0} l_{1} l_{2}}$ set of $2^{8}$ plaintext values $\left(\left(l_{0}, l_{1}, l_{2}, \lambda_{3}\right),\left(\rho_{0}, r_{1}, \rho_{2}, \rho_{3}\right)\right)$, where $\left(r_{1} \in[0.255]\right)$, thus obtaining $2^{8}$ encrypted values 
$\left(\left(l_{0}, l_{1}, l_{2}, l_{3}\right),\left(r r_{0}, r r_{1}, r r_{2}, r r_{3}\right)\right)$. (Note: this can be done once for all for all $c_{1} \oplus c_{3}$ assumptions).

- We encrypt the $X^{\prime} l_{0} l_{1} l_{2}$ set of $2^{8}$ plaintext values $\left(\left(l_{0}, l_{1}, l_{2}, \lambda_{3} \oplus \alpha\right),\left(\rho_{0} \oplus\right.\right.$ $\left.\left.\delta_{0}, r_{1}^{\prime}, \rho_{2} \oplus \delta_{2}, \rho_{3} \oplus \delta_{3}\right)\right)$ where $r_{1}^{\prime} \in[0.255]$; thus obtaining $2^{8}$ encrypted values $\left(\left(l_{0}^{\prime}, l_{1}^{\prime}, l l_{2}^{\prime}, l_{3}^{\prime}\right),\left(r r_{0}^{\prime}, r r_{1}^{\prime}, r r_{2}^{\prime}, r r_{3}^{\prime}\right)\right)$

- We now want to find all the (P, $\left.\mathrm{P}^{\prime}\right)$ pairs in $\left(\mathrm{X}_{l_{0} l_{1} l_{2}} \times \mathrm{X}^{\prime} l_{0} l_{1} l_{2}\right)$ such that $l_{2}=$ $l_{2}^{\prime} ; r r_{2}=r r_{2}^{\prime}$ (i.e. we want to "filter" those pairs of $\left(\mathrm{X}_{l_{0} l_{1} l_{2}} \times \mathrm{X}_{l_{0} l_{1} l_{2}}\right)$ for which the inputs and outputs of the S-boxes in the two last rounds of the encryption are equal), and group these pairs according to the $r_{1} \oplus r_{1}^{\prime}$ difference value. This can be done efficiently, in about $2^{8}$ operations in average, and provides in average one $\left(P, P^{\prime}\right)$ pair after filtering.

For each $\delta_{1}=r_{1} \oplus r_{1}^{\prime}$ candidate difference, we thus obtain, by merging the contributions obtained for each $l_{0} l_{1} l_{2}$ value, a list $S$ of $2^{16}$ (P, $\left.\mathrm{P}^{\prime}\right)$ pairs in average. Let us consider the $\Delta S$ set of output xors for the ( $\left.P, P^{\prime}\right)$ pairs contained in such a list :

\section{Claim :}

(i) : If the $\left(\delta_{0}, \delta_{1}, \delta_{2}, \delta_{3}\right)$ candidate is correct, then the $\Delta S$ differences are picked from a set of size less than $128^{\circ} / 256^{2}$.

(ii) : If the $\left(\delta_{0}, \delta_{1}, \delta_{2}, \delta_{3}\right)$ candidate is wrong, then the $\Delta S$ differences are picked from a set of size about $256^{6}$.

(iii) : The size of the $S$ sets (about $2^{16}$ pairs) is sufficient to test $\left(\delta_{0}, \delta_{1}, \delta_{2}, \delta_{3}\right)$, by comparing the sizes of $S$ and $\triangle S$, i.e. by counting how many collisions occur between the output xors for $S$ pairs.

Proof (heuristic arguments) : In the first case, $S$ is a subset of the $S_{\lambda_{3}, \alpha}$ considered in Proposition 2. Moreover, $\Delta S=\Delta S[14]$, because the two last rounds have no effect on the output xor of an $S$ pair. The $\Delta S[14]$ elements are picked from a set of size about $\left|\Delta S_{\lambda_{3}, \alpha}[14]\right| / 256^{2}$ because $\Delta S[14]$ contains only $\Delta S_{\lambda_{3}, \alpha}[14]$ elements $\left(\left(\delta_{l_{0}}, \delta_{l_{1}}, \delta_{l_{2}}, \delta_{l_{3}}\right),\left(\delta_{r_{0}}, \delta_{r_{1}}, \delta_{r_{2}}, \delta_{r_{3}}\right)\right)$ such that $\delta_{l_{2}}=0$ and $\delta_{r_{2}}=0$.

The first part of the claim now results from : $\left|\Delta S_{\lambda_{3}, \alpha}[8]\right|=1$ (by Proposition 2)

and

$\left|\Delta S_{\lambda_{3}, \alpha}[14]\right| \leq 128^{(i)}\left|\Delta S_{\lambda_{3}, \alpha}[8]\right|$ (by Proposition 3).

The second part of the claim follows from the assumption that in other cases, the final xor values for pairs in the $S$ set simply behave as random elements of the set of $\left(\left(\delta_{l_{0}}, \delta_{l_{1}}, \delta_{l_{2}}, \delta_{l_{3}}\right),\left(\delta_{r_{0}}, \delta_{r_{1}}, \delta_{r_{2}}, \delta_{r_{3}}\right)\right)$ differences such that $\delta_{l_{2}}=0$ and $\delta_{r_{2}}=0$. 
The third part of the claim follows from the facts that since $2^{16} \gg\left(128^{6} / 256^{2}\right)^{1 / 2}$ many collisions will occur in the first case, whereas few collisions will occur in the second case, since $2^{16} \ll\left(256^{6}\right)^{1 / 2}$.

Because of the difference between behaviours (i) and (ii), Substep 3.1 provides $c_{1} \oplus c_{3}$, (as the value for which one $\delta_{1}$ value behaves according to (i) ), and also the $\delta_{0} \delta_{1} \delta_{2} \delta_{3}$ difference for the considered $\alpha \neq 0$ value. Substep 3.1 requires to encrypt about $2^{40}$ chosen plaintexts, and abont $2^{40}$ operations.

Substep 3.2 : We are doing the same search as at Substep 3.1 for each of the 254 remaining $\alpha \neq 0$ values, but the $c_{1} \oplus c_{3}$ byte has no longer to be exhaustively searched, since it has been determined at Substep 3.1.

Step 3 requires to encrypt about $2^{41}$ plaintext. blocks, and the computational cost of Step 3 is about $2^{41}$ operations. After Step 3 , the $p_{0}, p_{1}, p_{2}$ and $p_{3}$ permutations are entirely determined, up to the five unknown bytes $c_{3}, p_{0}\left[c_{3}\right], p_{1}\left[c_{3}\right]$, $p_{2}\left[c_{3}\right]$, and $p_{3}\left[c_{3}\right]$, and the output of round 1 is known up to 8 constant bytes.

Step 4 is based on the results of Step 3 .

We define four permutations $\bar{p}_{0}, \bar{p}_{1}, \bar{p}_{2}, \bar{p}_{3}$, by the relations:

$\left(\bar{p}_{0}, \bar{p}_{1}, \bar{p}_{2}, \bar{p}_{3}\right)\left[\lambda_{3}\right]=0$

$\left(\bar{p}_{0}, \bar{p}_{1}, \bar{p}_{2}, \bar{p}_{3}\right)\left[\lambda_{3} \oplus \alpha\right]=\left(\delta_{0} ; \delta_{1}, \delta_{2}, \delta_{3}\right)(\alpha)$ for $\alpha \neq 0$.

We thus define a $\left[\bar{p}_{0}, \bar{p}_{1}, \bar{p}_{2}, \bar{p}_{3}\right] \mathrm{S}$-box which is intended to be the basis for the construction of an equivalent representation of the first half of Khufu.

If we set $\overline{K 1}_{3}=0$, the assumptions $\left[p_{0}, p_{1}, p_{2}, p_{3}\right]=\left[\bar{p}_{0}, \bar{p}_{1}, \bar{p}_{2}, \bar{p}_{3}\right]$ and $K 1_{3}=$ $\overline{K 1}_{3}$ provide the output of round 1 up to 8 unknown constant bytes for every input block.

The purpose of Step 4 is to gradually derive, one after the other, seven additional bytes $\overline{\kappa 2}_{3}, \overline{K 1}_{1}, \overline{K 2}_{1}, \overline{K 1}_{0}, \overline{\kappa 2}_{0}, \overline{\kappa 1}_{2}, \overline{\kappa 2}_{2}$ such that :

- the assumptions $\left[p_{0}, p_{1}, p_{2}, p_{3}\right]=\left[\bar{p}_{0}, \bar{p}_{1}, \bar{p}_{2}, \bar{p}_{3}\right], K 1_{3}=\overline{K 1}_{3} ; K 2_{3}=\overline{K 2}_{3}$ provide the output of round 2 up to 8 unknown constant bytes for every input block;

- the assumptions $\left[p_{0}, p_{1}, p_{2}, p_{3}\right]=\left[\bar{p}_{0}, \bar{p}_{1}, \bar{p}_{2}, \bar{p}_{3}\right] ; K 1_{3}=\bar{\kappa}_{3} ; \kappa 2_{3}=\overline{\kappa 2}_{3}$; $K 1_{1}=\overline{K 1}_{1} ; K 2_{1}=\overline{\kappa 2}_{1} ; K 1_{0}=\bar{\kappa}_{0} ; K 2_{0}=\overline{\kappa 2_{0}} ; K 1_{2}=\bar{\kappa}_{2} ; \kappa 2_{2}=\bar{\kappa}_{2}$ provide the output of round 8 up to 8 unknown constant bytes for every input block.

Our estimate of the cost of the derivation of the above cquivalent hey bytes is at most $2^{40}$ plaintext blocks per key byte $\left(2^{32}\right.$ per test of an assumption on such a byte), using differential techniques similar to the one of Proposition 2 : introduction of a fixed difference in the $S$-box inputs of round $r$ and compensation of the resulting difference for the $S$-box inputs of the subsequent rounds until round 8 , with $r=1$ in Proposition 2 and $r=2$ to 8 here. Once the seven 
above equivalent key bytes have been derived, a differential attack on the second half of Khufu (i.e. rounds 8 to 16 and the final xor with auxiliary key bits) can be mounted, at no substantial additional expense.

\section{Aknowledgements}

The attack presented in this paper is based on a preliminary investigation of Khufu in cooperation with Sean Murphy.

We also want to thank Thierry Baritaud for his help in the elaboration of the Latex version of this paper.

\section{Conclusion}

We have shown in some detail that first information concerning the secret $\mathrm{S}$ box used in the first half of the scheme can be derived with about $2^{31}$ chosen plaintexts, and about $2^{31}$ operations (Step 1). Our estimate of the resources required for breaking the whole scheme is about $2^{43}$ chosen plaintexts and $2^{43}$ operations (Steps 2 to 4 ). However, further verifications (in particular computer experiments) are required to make sure that the figures announced in the description of Stcps 3 and 4 are valid. Although the proposed attack is far from being realistic, becanse of the required amount of chosen plaintext, it suggests that the security of the 16-round Khufu might be too low for providing a suitable replacement to DES. In faimess to Merkle, it should be noticed however that some warnings concerning the choice of the 16-round version as compared with the 24-round and 32-round versions are already contained in [1], in particular the remark that the "safety factor" in Khufu with 16 rounds is less than that of DES.

We have found no similar attack for the 24-round version of Khufu.

\section{References}

1. Ralph Merkle, "Fast Software Fncryption Functions", Advances in Cryptology Crypto'90, Spriuger Verlag.

2. E. Biham and A. Shamir. "Differential Cryptanalysis of Snefru, Khafre, REDOCII, LOKI, and Lucifer". Aclvances in Cryptology - Crypto'91, Springer Verlag.

3. E. Biham and A. Shamir, "Differential Cryptanalysis of the Data Encryption Standart". Springer Verlag, 1993, Chapter 7. 